\title{
Traffic Shaping Trends in Optical Packet/Burst Switching Networks
}

\author{
Maria-Dolores Cano, Pablo Pavon-Mariño, Alejandro Ortuño-Manzanera, Joan Garcia-Haro \\ Department of Information Technologies and Communications \\ Technical University of Cartagena (UPCT) \\ 30202 Cartagena, Spain \\ e-mail: \{mdolores.cano,pablo.pavon,joang.haro\}@upct.es
}

\begin{abstract}
This paper surveys a set of relevant techniques proposed for traffic shaping in backbone OBS/OPS networks. The work is intended to fuel the near future research in the topic the authors will conduct within BONE project.
\end{abstract}

Keywords: traffic shaping, traffic conditioning, OPS, OBS.

\section{INTRODUCTION}

It is known that Internet traffic exhibits a structural self-similarity over a wide range of time scales. Existing performance evaluation studies in Optical Packet Switching (OPS) and Optical Burst Switching (OBS) networks show high contention performance degradation in the nodes under correlated traffic [1]. The reason lies on the adverse effect over the contention resolution schemes. As an example, for an OPS node able to emulate output buffering, operating with fixed size packets which are aligned at switch inputs, 2 input and output fibres, and 32 wavelengths per fibre, 2 Fibre Delay Lines (FDLs) are enough to achieve a $10^{-8}$ packet loss probability under uncorrelated traffic. However, on-off input traffic with moderate burst sizes increases the buffering requirements to $10 \mathrm{FDLs}$ (which implies $9 \mathrm{~km}$ of fibre, if packet duration of $1 \mu \mathrm{s}$ is assumed) just for reaching a loss probability of $10^{-5}$. We can not blame the switching architecture for this performance degradation, as an optimum switching fabric able to emulate output buffering is used. Therefore, in order to make OPS/OBS technology feasible, the loss performance problem must be solved from acting in other parts of the network. The authors believe that traffic shaping at the network edge is the right place to apply techniques that smooth traffic injection. This paper surveys a set of techniques proposed in the field.

The rest of the paper is organized as follows. Section 2 collects and describes main proposals in the field. Section 3 concludes the paper.

\section{TRAFFIC CONDITIONING IN OPS}

One way to reduce burstiness of input traffic, and consequently the effects of self-similar traffic, is traffic conditioning at the edge node. In order to classify traffic conditioning methods for optical switched networks we can use several approaches (see Table 1). In this section, we describe the operation of these methods and summarize their performance results. It is interesting to note that methods where not compared among themselves in any work, leaving a blank comparative that could bring appealing results.

Table 1. Comparing traffic conditioning techniques in Optical Switching Networks.

\begin{tabular}{|l|l|c|c|c|}
\hline \multicolumn{1}{|c|}{ Type } & \multicolumn{1}{|c|}{ Name } & Where & OBS/OPS & Nodes simulated \\
\hline \multirow{2}{*}{$\begin{array}{l}\text { Based on maximum } \\
\text { optical size and maximum } \\
\text { aggregation delay }\end{array}$} & $(\psi-\tau)$-Scheduler/Shaper & Assembling & OBS & Multi-node \\
\cline { 2 - 5 } & OPORON & Assembling & A-OPS & Mono-node \\
\cline { 2 - 5 } \\
$\begin{array}{l}\text { Traffic shaping with fixed } \\
\text { output rate }\end{array}$ & Classical shaping & $\begin{array}{c}\text { Aptical packet } \\
\text { transmission }\end{array}$ & S-OPS & Multi-node \\
\hline \multirow{2}{*}{$\begin{array}{l}\text { Traffic shaping with } \\
\text { variable output rates }\end{array}$} & $\begin{array}{l}\text { Shaping with variable rates } \\
\text { and delay restrictions }\end{array}$ & $\begin{array}{c}\text { Optical packet } \\
\text { transmission }\end{array}$ & S-OPS & Mono-node \\
\cline { 2 - 5 } & $\begin{array}{l}\text { Traffic shaping with } \\
\text { renegotiation }\end{array}$ & $\begin{array}{c}\text { Optical packet } \\
\text { transmission }\end{array}$ & S-OPS & Multi-node \\
\hline
\end{tabular}

\subsection{Based on maximum optical size and maximum aggregation delay}

In this section, we present a common technique to improve statistical characteristics of incoming optical traffic, i.e. the optical traffic injected into the core optical network. The idea can be summarized as follows. Ingress electronic IP traffic at the edge nodes is conditioned during assembling using two parameters: i) the maximum size of the optical packet (or the optical burst), and ii) the maximum time that an IP packet can remain in the buffer without being aggregate into an optical packet (or burst) that leaves the buffer. In general terms, these proposals agree that the bigger the optical packet (burst) size, the smaller the probability of optical packet (burst) loss, but this results in a longer traffic delay. As an example of this approach we briefly describe the $(\psi-\tau)$ Scheduler/Shaper for optical coarse packet switching IP-over-WDM networks introduced by [2], the class-based 
system for asynchronous OPS networks introduced in the OPORON (Optical Packet switching Over wavelength ROuted Network) project [3], and the traffic shaping method presented in [4].

\subsubsection{The $(\psi-\tau)$-Scheduler/Shaper}

In this section, we explain the $(\psi-\tau)$-Scheduler/Shaper [2] for OBS networks, where $\psi$ is the maximum burst size and $\tau$ is the maximum assembly time for an optical burst. It is a QoS enhanced traffic control scheme used during packet aggregation at ingress nodes, aiming at providing delay and loss class differentiation for Optical Coarse Packet Switching (OCPS). It is assumed that IP packet flows are designated with delay-associated weights. Although in the paper is not specified how these weights are set, they could be inferred from an MPLS tag or a DiffServ value. Then, to provide delay class differentiation, the scheduler performs packet scheduling and assembly into bursts based on their weights and the maximum burst size $\psi$. The operation is described in Fig. 1.a). We observe that in an ingress node, incoming packets are first classified based on destination/loss classes, from $\mathrm{C}_{1}$ to $\mathrm{C}_{\mathrm{M}}$. It is assumed that packets with the same destination and the same loss probability level use the same Optical Label Switch Path (OLSP). Notice that with this scheme, an optical burst may contain packets from up to $N$ different delay classes (from $\mathrm{C}_{\mathrm{m}, 1}$ to $\mathrm{C}_{\mathrm{m}, \mathrm{N}}$ ). Within the same destination/loss class $\mathrm{C}_{\mathrm{m}}$, the scheduler/shaper is responsible for selecting, according to the pre-assigned delay weights, the order in which packets from queues $\mathrm{C}_{\mathrm{m}, 1}$ to $\mathrm{C}_{\mathrm{m}, \mathrm{N}}$ are inserted into the optical burst. The higher the priority of a delay class, the more packets of that delay are allowed into the optical burst. Second, to provide loss class differentiation the shaper performs traffic shaping with larger burst sizes assigned to higher loss priority classes, i.e. using a different maximum burst size $\psi$ for each class $\mathrm{C}_{\mathrm{m}}$.

An entire optical network, with QoS burst truncation and full wavelength conversion capabilities in each switching node, is simulated to evaluate burstiness and loss probability differentiation. Authors demonstrate that the $(\psi-\tau)$-Scheduler/Shaper assures low delay/bound for high delay priority classes at a cost of increasing delay/bound for low delay priority classes. However, given parameters $\psi$ and $\tau$, authors achieved meeting a delay class delay/bound requirements by appropriately tuning the weight of a delay class. In addition, results show that the greater the $\psi$ value, the lower the burst loss probability. Comparing the performance of JET (Just Enough Time)-based OBS with the authors' proposal (OCPS with the ( $\psi-\tau)$-Scheduler/Shaper), the loss probability decreases up to five orders of magnitude.

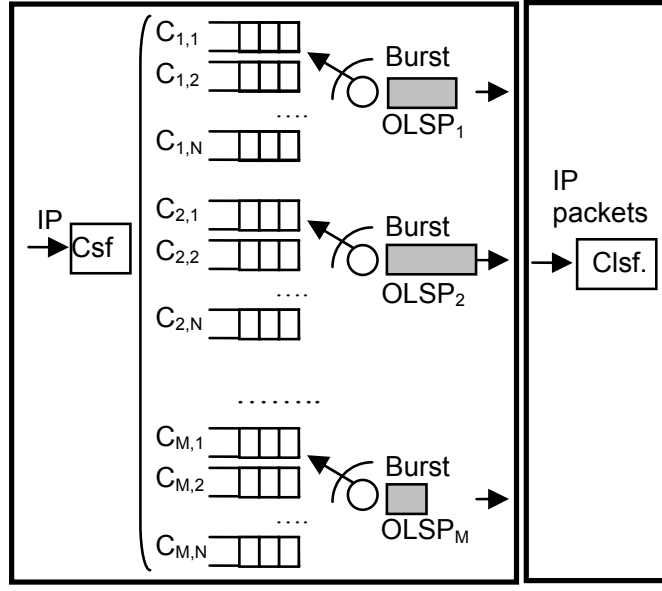

a) $\lceil 2\rceil$

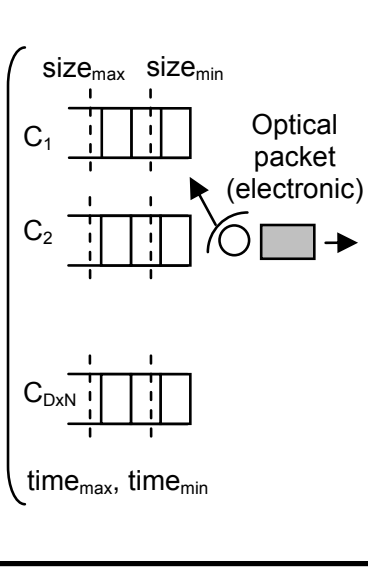

b) $\lceil 3\rceil$

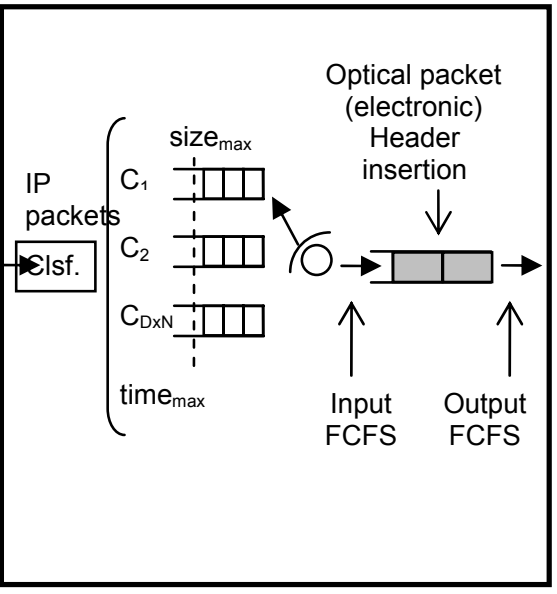

c) $\lceil 4\rceil$

Figure 1. a) ( $\psi-\tau)$-Scheduler/Shaper system architecture; b) OPORON class-based traffic shaping; c) Proposal for synchronous OPS networks adding a FCFS queue to the Fig. 1 b).

\subsubsection{The OPORON approach}

In this section, we explained the class-based traffic aggregation in the hybrid IP-Optical packet-switched edge router proposed in [3] for an edge node in an OPS asynchronous network with variable length for optical packets. The edge router with traffic shaping capabilities provides mapping between the electronic and optical domains through aggregation of the electronic packets, according to the destination address and Class of Service $(\mathrm{CoS})$, into an optical packet payload with appropriate optical label and wavelength. The CoS aggregation method, where traffic shaping takes place, works as follows (Fig. 1.b)). The system architecture has as many queues (aggregation buffers) as CoS per destination addresses. Each aggregation buffer has two parameters for holding time (maximum and minimum) and two parameters for optical packet size (maximum and minimum) that determine when an optical packet is created. When an IP packet arrives, it is checked if there is room at its corresponding buffer based on $\mathrm{CoS}$ and destination address. If there is no room, then a buffer corresponding to a higher $\mathrm{CoS}$ and same destination address is checked. If all buffers are busy, the buffer that corresponds to the packet is emptied regardless of the previous parameters. Then, the optical packet is created and sent. Once the IP 
packet has been added to a buffer, the aggregation parameters are checked, both time and size, searching for the trigger zone conditions. If conditions are satisfied, i.e. the current aggregation time is between the minimum and maximum holding times, and the current aggregated packet size is within the minimum and maximum packet size, then the optical packet is created and sent.

The performance of the algorithm is studied through simulation in a one-node topology and compared with the classical aggregation method. The parameters for the trigger zone are obtained from the observation of the input IP traffic profile, generated by a multifractal wavelet model. Results show that self-similarity is slightly reduced, from a Hurst parameter $(\mathrm{H})$ with value 0.8 to $\mathrm{H}$ equal to 0.6 (classical method) and 0.5 (proposed method). However, the mean aggregation delay is reduced from $4.8 \mu \mathrm{s}$ (classical method) to $3.1 \mu \mathrm{s}$ (proposed method), i.e. a reduction of $30 \%$.

\subsubsection{Traffic shaping with a FCFS system}

The study done in [4] by simulation shows the behavior of three statistics (long-range dependence -LRD-, short-range dependence -SRD-, and marginal distribution), in an edge node of a synchronous OPS network that performs traffic shaping to ingress traffic. Two tasks are done at the edge node, called in this case assembling and header insertion. Assembling is very similar to the method proposed in section 2.1 .2 and is shown in Fig.1 c). Main difference is that after classification and assembling, optical packets in electronic format are stored in a FCFS (First Come First Serve) queue for header insertion. Input and output traffics to/from the FCFS queue are sampled to analyze statistics under a network load of 0.3, 0.5, and 0.8. Incoming self-similar IP traffic is modeled by the superposition of several fractal renewal processes (Sup-FRP). The IP packet distribution is addressed by using four different sizes $(40 \mathrm{~B}, 44 \mathrm{~B}, 576 \mathrm{~B}, 1500 \mathrm{~B})$ with their corresponding probabilities, having predominance of small IP packets. Regarding the LRD, evaluated using the Hurst parameter $(\mathrm{H})$, the authors obtained the following results. For a low network load, an increment in the optical packet size or an increment in the maximum time inside the queue means an increment in $\mathrm{H}$ for both traffics: input and output to/from the FCFS queue. Compared to ingress IP traffic, the reduction of $\mathrm{H}$ is around 0.1 , as it occurred with the OPORON approach. Therefore, the FCFS strategy has a negligible effect on H. For a medium load applies the same results. For a heavy load, there is a drastically reduction in $\mathrm{H}$, so the schedule queue FCFS makes the traffic smoother to reduce the bursty degree, what simultaneously introduces longer delays for IP packets and a smaller loss probability. Concerning SRD and marginal distribution, the FCFS has no effect on them comparing input/output FCFS traffic, but they decrease compared to incoming IP traffic.

\subsection{Classical traffic shaping to aggregated optical packets}

In this section, we depict the classical shaping. Instead of achieving smoother traffic via some technique at packet assembling, these authors apply traffic shaping over the optical packets in electronic format. The shaping proposed in [5] is quite simple and it is proposed for a synchronous slotted OPS network with fixed packet sizes. Optical packets (always in electronic format) are shaped before entering into the optical domain. An ingress node is assumed to have as many queues for optical packets as destinations (egress ports). For several even spacing values, authors demonstrate that packet loss due to contention is minimized. Nevertheless, as we have seen in other works previously mentioned, it also represents a higher delay. Simulations show that a delay of the order of hundred of $\mu$ s could be reached if an FDL with capacity 20. For a more viable FDL [1] of 5 positions, shaping should be more aggressive (more spaced) and consequently delays could be substantial.

\subsection{Traffic shaping with variable output rates}

In this section, we describe two works that tackled the conditioning problem from a different perspective. As done in the previous section, traffic conditioning is done with optical packets in the electronic domain, before entering the optical core network. However, the result of applying a shaper over the optical packets (electronic format) will not be a constant packet rate, but a variable one depending on several traffic or network performance parameters.

\subsubsection{Traffic shaping to aggregated optical packets with variable output rates and time restrictions}

To overcome the drawback of the delay introduced by shaping traffic, authors in [6] propose to smooth traffic in a way that the output shaped rate may be variable but the delay is limited. In other words, disregard of incoming traffic, outgoing traffic is as smooth as possible not to exceed a time constrain (see Fig. 2). This idea was taken from previous works on traffic conditioners for video transmission, called traffic conditioning off-line. Authors modified this traffic conditioning off-line to make it independent of the traffic profile, i.e. so that it can work online. The proposal is evaluated through simulation and shows a notably reduction in loss probability due to contention at the core node keeping the time restrictions.

\subsubsection{Traffic shaping with renegotiation}

Following the same trend, Lu et al. present in [7] a queuing and scheduling method to shape user traffic at the edge nodes, generating a different fixed rate reliant on network conditions and user requirements. The idea is also taken from video transmission online techniques, but adapted to wide area networks characteristics. Each 
node should get relevant network information from other nodes by means of a control protocol, and should use this information to assess the best output rate that minimizes loss probability in core nodes due to contention. Renegotiation, i.e. to estimate the most appropriate output rate, will be done at regular intervals. Observe that this shaping is done per stream, where a stream is represented by a unidirectional communication between two edge nodes that need to communicate.

The algorithm proceeds as follows. If the requested service rate for next interval, obtained taking the mean of the previous intervals is bigger than the current service rate plus a small admitted quantity, then renegotiation is requested. If there is available bandwidth, the new output rate is calculated taking into account both user requirements and network conditions. The weight of each parameter is configurable. Otherwise, the more congested the network is, the more dependant the new output rate is to network conditions. Once traffic streams are shaped, if there is contention within the streams that leave the edge node, a scheduling is applied. This scheduling gives priority to streams with higher rate streams. All nodes in the network carry out the same scheduling criteria among all ongoing streams. Results show that the longer the edge rate negotiation interval, the more delay introduced in IP traffic but the smaller loss probability. Compared to a network that has neither traffic shaping nor scheduling, the proposal reduces loss probability by several orders of magnitude. Regarding scalability issues, authors mentioned the option of partitioning the network into smaller areas.
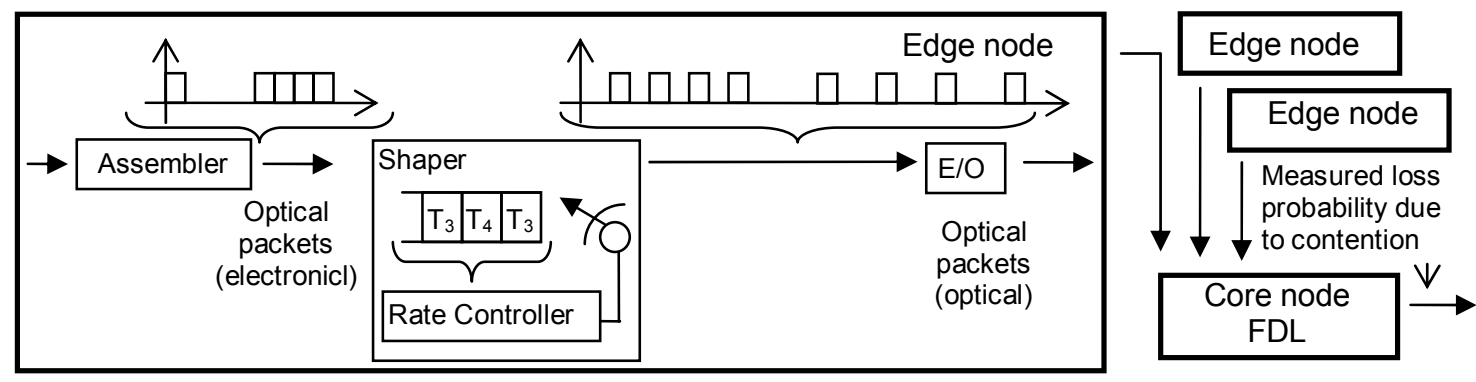

Figure 2. Traffic shaping with variable output rates and time [6].

\section{CONCLUSIONS}

This paper surveys several approaches for traffic shaping in OPS/OBS networks. The techniques can be categorized in assembler techniques, and transmission techniques. Both of them can be combined in the node. This work is intended to fuel further research in the topic, which will study combined techniques in a networkwide scenario.

\section{ACKNOWLEDGEMENTS}

This research has been supported by the BONE-project ("Building the Future Optical Network in Europe"), a Network of Excellence funded by the European Commission through the 7th ICT-Framework Program, the support of MEC project TEC2007-67966-01/TCM CON-PARTE-1, and it is also developed in the framework of "Programa de Ayudas a Grupos de Excelencia de la Región de Murcia, de la Fundación Séneca, Agencia de Ciencia y Tecnología de la RM (Plan Regional de Ciencia y Tecnología 2007/2010).

\section{REFERENCES}

[1] J. Veiga-Gontán, P. Pavón-Mariño, M. Izal, D. Morato, J. García-Haro, "Performance evaluation of slotted OPS switching fabrics under self-similar traffic", Proceedings of the 12th International Conference on Optical Networking Design and Modeling - ONDM 2008, Vilanova i la Geltrú (Spain), March 2008.

[2] M. C. Yuang, P.-L. Tien, J. Shih, A. Chen, "QoS Scheduler/Shaper for Optical Coarse Packet Switching IP-Over-WDM Networks", IEEE Journal on Selected Areas in Communications, vol. 22, no. 9, pp. 1766-1780, November 2004.

[3] R. Nejabati, G. Zervas, D. Simeonidou, M. J. O’Mahony, D. Klonidis, “The OPORON Project: Demonstration of a Fully Functional End-to-End Asynchronous Optical Packet-Switched Network", Journal of Lightwave Technology, vol. 25, no. 11, pp. 3495-3510, November 2007.

[4] D. Zhang, M. Zhang, P. Ye, "Traffic Shaping at the edge node in synchronous optical packet switched networks", Photonics Networks Communications, vol. 13, pp. 103-110, 2007.

[5] V. Sivaraman, D. Moreland, D. Ostry, "Ingress traffic conditioning in slotted optical packet switched networks", ATNAC 2004, pp. 162-169, Sydney, Australia, Dec 2004.

[6] V. Sivaraman, D. Moreland, D. Ostry, "A Novel Delay-Bounded Traffic Conditioner for Optical Edge Switches", Proc. IEEE HPSR'05, pp. 182-186, Hong Kong, May 2005.

[7] Z. Lu, D. K. Hunter, "Contention Reduction in Core Optical Packet Switches Through Electronic Traffic Smoothing and Scheduling at the Network Edge", Journal of Lightwave Technology, vol. 24, no.12, pp. 4828-4837, December 2006. 